\title{
Multiservice capacity and interference statistics of the uplink of high altitude platforms (HAPs) for asynchronous and synchronous WCDMA system
}

\author{
Bazil Taha Ahmed • Miguel Calvo Ramón
}

\begin{abstract}
In this work, the capacity and the interference statistics of the uplink of high-altitude platforms (HAPs) for asynchronous and synchronous WCDMA system assuming finite transmission power and imperfect power control are studied. Propagation loss used to calculate the received signal power is due to the distance, shadowing, and wall insertion loss. The uplink capacity for 3- and 3.75-G services is given for different cell radius assuming outdoor and indoor voice users only, data users only and a combination of the two services. For 37 macrocells HAP, the total uplink capacity is 3,034 outdoor voice users or 444 outdoor data users. When one or more than one user is an indoor user, the uplink capacity is 2,923 voice users or 444 data users when the walls entry loss is $10 \mathrm{~dB}$. It is shown that the effect of the adjacent channels interference is very small.
\end{abstract}

Keywords Uplink capacity · HAPs · Power control · WCDMA · Walls entry loss · Walls penetration loss · HSUPA

\section{Introduction}

There is an insatiable demand for communications services throughout the world, driven largely by the need for Internet access. Wireless offers the only viable provision means in many scenarios, but both terrestrial and satellite systems suffer from fundamental limitations in cost and capacity.
One potential delivery method is from high-altitude platforms (HAPs), which are pilotless solar-powered airships or aircraft operating at an altitude between 17 and $24 \mathrm{~km}$ due to the reduced wind speed. A HAP system may be viewed as either a very low-altitude stationary satellite or a very tall radio mast, and can offer communications services with the best features of both.

Airship technology is developing steadily, with commercial applications becoming more of a reality. Wireless communication from HAPs offers considerable potential for new broadband services, for mobile phones and for rich markets such as disaster relief or military where rapid deployment is a key feature.

In UMTS systems, power control of the user's transmitted power is get using the open loop and closed-loop power control. The open loop power control is the ability of the user equipment (UE) transmitter to set its output power to a specific value. It is used for setting initial uplink and downlink transmission powers when a UE is accessing the network. The open loop power control tolerance is $\pm 9 \mathrm{~dB}$ (normal conditions) or $\pm 12 \mathrm{~dB}$ (extreme conditions). Closed-loop power control procedures in UMTS are standardized by 3 GPP. The closed-loop power control can be further divided into two processes: outer-loop power control and inner-loop power control (also called fast closed-loop power control). The inner-loop power control in the uplink is the ability of the UE transmitter to adjust its output power in accordance with one or more transmit power control (TPC) commands received in the downlink, in order to keep the received uplink signal-to-interference ratio (SIR) at a given SIR target. The UE transmitter is capable of changing the output power with a step size of 1,2 and $3 \mathrm{~dB}$, in the slot immediately after the TPC command can be derived. Innerloop power control frequency is $1,500 \mathrm{~Hz}$. Perfect power control (where SIR of all users is the same) can be get using 
a very small power control step dealing with users with very low mobility (causing very small propagation loss change). It is obvious that it is impossible to comply both of the two above given requirement. In practice, the SIR of the uplink users is normally distributed around the mean value giving a rise to the imperfect power control. The error in the power control and in consequence the standard deviation of the power control error increases in general with the increment of the power control step and the mobility (speed) of the UMTS users.

Wireless communications using HAPS have been proposed worldwide due to the many advantage of HAPS system over terrestrial tower-based and satellite systems [1]. Recently, it has been accepted to use HAPS as an alternative means to deliver the third generation IMT-2000 wireless services.

Downlink power control is used in cellular WCDMA systems to equalize the SNR ratio of the users within the cell. The effect of this strategy is the reduction of the interference to the other neighboring cells and the increment of the downlink capacity. The performance of a distance based power control scheme based on an $n$th exponent of mobile's distance from the center of its serving cell was studied in [2] and used to analyze the downlink capacity of the high altitude platform WCDMA system in [3] (for voice service only). In [4], the HAPs downlink capacity has been analyzed using a new power control scheme. In [5], the uplink capacity of HAP has been analyzed assuming perfect power control. Nothing about the interference variance was mentioned in [5]. Thus, the given uplink capacity was the maximum capacity. In [6] and [7], the uplink capacity of mulitservice WCDMA HAP systems has been calculated without taking into account the variance of the interference. Thus, the given uplink capacity was the maximum capacity. Results have been given assuming only a single radius corresponding to an antenna gain of $-10 \mathrm{~dB}$ lower than the maximum antenna gain of $35 \mathrm{dBi}$. This means that the HAP antenna gain is $25 \mathrm{dBi}$ for users at the cell edge.

In [8], the uplink capacity has been computed for a single service system for imperfect power control. Here also, nothing about the interference variance was mentioned, thus, the given uplink capacity represents an optimistic uplink capacity.

In [9], Hong et al. have studied the capacity of a WCDMA system using high-altitude platform stations where nothing about the interference variance has been mentioned. Results have been given assuming only single radius corresponding to an antenna gain of $-13 \mathrm{~dB}$ form the maximum antenna gain of $35 \mathrm{~dB}$

A HAPs carrying a WCDMA communication payload and a multibeam-phased array antenna with beam/gain shaping capability positioned at altitude $(h)$ of $22 \mathrm{~km}$ was proposed in [3]. The receiving antenna radiation pattern is depieted in Fig. 1 where the maximum gain is $37 \mathrm{dBi}$.
In this work, the multiservice uplink capacity and the interference statistics (expected value and variance) of asynchronous and synchronous WCDMA will be investigated assuming finite transmitted power and imperfect power control. Also, the uplink capacity will be given for different cell radius and different services such as voice, traditional data, and high-speed uplink packet access (HSUPA) when the outage probability is $1 \%$ where the uplink capacity is studied assuming an outdoor and indoor users something that is investigated for the first time.

\section{HAP uplink capacity}

In this section, we will study the uplink capacity and interference statistics assuming finite transmission power and imperfect power control. In this work, capacity is defined as the maximum number of simultaneous users that the UMTS system can support for a given outage probability.

For a given service $s$, let us assume that the expected value of the power level of the desired signal received by the HAP receiver is $P_{r, s}$. Then, for an imperfect power control, the expected value of the intracellular interference power $E$ $[P]_{\text {in,s }}$ due to users of the cell under study is given by:

$E[P]_{\mathrm{in}, s} \approx e^{\left(\beta^{2} \sigma_{c}^{2} / 2\right)}(1-\phi) \alpha_{s} N_{u, s} P_{r, s}$

Where $\beta=(\ln 10) / 10$ and $\sigma_{\mathrm{c}}$ is the standard deviation of the power control error. $\phi$ is the orthogonality factor of the uplink assumed to be 0 for asynchronous WCDMA meanwhile it has a value of 0.5 or even higher for synchronous WCDMA [10], $\alpha_{s}$ is the source activity factor of the users of the service $s$ and $N_{u, s}$ is the number of the users of the service $\mathrm{s}$. Here, we assume that the intracellular interference is a Gaussian process (has a normal distribution) [11].

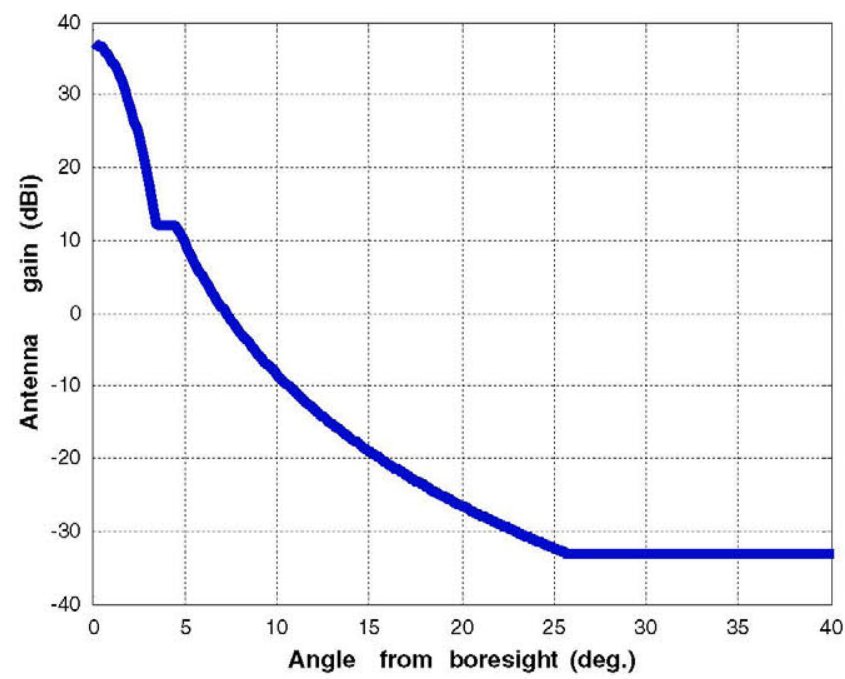

Fig. 1 Radiation pattern of the HAPs antenna 
The HAPs interference geometry is shown in Fig. 2. Assuming that $(i, j)$ denotes the $i$ th mobile in the $j$ th interfering cell and $\mathrm{BS}_{j}$ denotes the base station serving the $j$ th interfering cell. Mobile $(i, j)$ located at a distance $r_{i j}$ away from the center of its serving cell and $r_{o, i, j}$ from the center of the cell under study. The transmitted power of the mobile ( $i$, j) power controlled by $\mathrm{BS}_{j}$ is given by:

$$
P_{T, i j, s}=P_{r, s} l_{i j}^{\prime} 10^{-\zeta_{i j} / 10} 10^{-G\left(\psi_{i j}\right) / 10}
$$

This mobile produces an interference power at $\mathrm{BS}_{o}$ given as:

$I_{B o}=e^{\left(\beta^{2} \sigma_{c}^{2} / 2\right)} P_{r, s}\left(\frac{l_{i j}}{l_{o, i j}}\right)^{\gamma} 10^{\frac{\left(\zeta_{o, i j}-\zeta_{i j}\right)}{10}} 10^{\frac{G\left(\psi_{o, i j}\right)-G\left(\psi_{i j}\right)}{10}}$

Where

- $l_{i j}$ and $l_{o, i j}$ are the distances from the mobile to $\mathrm{BS}_{j}$ and $\mathrm{BS}_{o}$, respectively;

- $\zeta_{i j}$ and $\zeta_{o, i j}$ denote the shadowing in decibels corresponding to these two paths;

- $\gamma$ is the path loss exponent $=2$;

- $G\left(\psi_{i j}\right)$ and $G\left(\psi_{o, i j}\right)$ are the normalized receiving antenna gains in decibels evaluated at the angles under which the mobile is seen from $\mathrm{BS}_{j}$ and $\mathrm{BS}_{o}$, respectively.

Due to the unique HAPS geometry, the transmit antenna beams of all macrocells essentially originate from the same point [3], so $l_{i j}=l_{o, i j}$ and $\zeta_{i j}=\zeta_{o, i j}$ (total correlation). Thus

$I_{B o} \approx e^{\left(\beta^{2} \sigma_{c}^{2} / 2\right)} P_{r, s} 10^{\frac{G\left(\psi_{o}, i j\right)-G\left(y_{i j}\right)}{10}}$

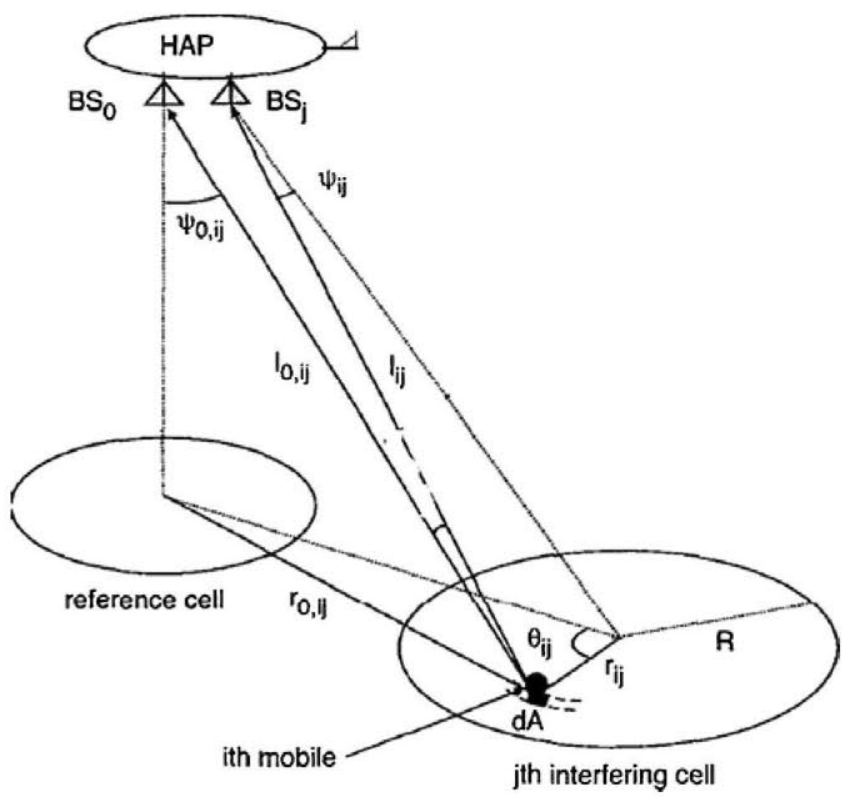

Fig. 2 HAP interference geometry
For the service $\mathrm{s}$, the expected value of the intercellular interference $E[P]_{\text {out }, s}$ due to $\left(N_{\text {cells }}-1\right)$ cells around the cell under consideration is given by:

$E[P]_{\mathrm{out}, s}=\alpha_{s} e^{\left(\beta^{2} \sigma_{c}^{2} / 2\right)} P_{r, s} N_{u, s} \sum_{j=1}^{N_{\text {cells }}-1} \int_{A} 10 \frac{\frac{G\left(\psi_{o, i j}\right)-G\left(\psi_{i j}\right)}{10}}{10} d A$

$=\alpha_{s} e^{\left(\beta^{2} \sigma_{c}^{2} / 2\right)} P_{r, s} N_{u, s} \sum_{j=1}^{N_{\text {cells }}-1} \int_{0}^{2 \pi} \int_{0}^{R} 10^{\left.\frac{G\left(\psi_{o, i}\right)}{10}\right)} \frac{G\left(\psi_{i j}\right)}{\pi R^{2}} r_{i j} d r_{i j} d \theta_{i j}$

$=\alpha_{s} e^{\left(\beta^{2} \sigma_{c}^{2} / 2\right)} N_{u, s} P_{r, s} F_{u}$

where $F_{u}$ is the other cells interference factor. Here, we also assume that the intercellular interference is a Gaussian process (has a normal distribution) [11].

The HAP main receiver consists of $N$ parallel subreceivers each one is assigned to serve a given macrocell treating the signal of other macrocells as a noise. Furthermore, each subreceiver is divided in parallel blocks each one is used to process a given service treating the signal of the other services as a noise. Each block is divided in parallel circuits each one of them is used to detect the signal of a given user treating the signal of other users of the same service as a noise. This topology that can be defined as a parallel uncorrelated detection process generates a statistical independence among macrocells and services from the interference point of view. The receiver management circuit is used to get the maximum possible system (represented by the users of $N$ macrocells) throughput controlling the load of each macrocell.

The expected value of the uplink total interference $E[P]_{t}$ is given by:

$E[P]_{t, s}=\left(E[P]_{i n, s}+E[P]_{o u t, s}\right)$

When $M$ services are considered, then the expected value of the total interference power $E[P]_{t}$, is given by:

$E[P]_{t}=\sum_{s=1}^{M} E[P]_{t, s}$

From [12] and taking into account our special case, the variance of the intracellular interference power of the services $s$ is calculated as:

$\operatorname{var}[P]_{i n, s} \approx(1-\phi) N_{u, s} P_{r, s}^{2}\left(p \alpha_{s}-q \alpha_{s}^{2}\right)$ 
Where

$p=e^{2 \beta^{2} \sigma_{c}^{2}}$

$q=e^{\beta^{2} \sigma_{c}^{2}}$

The variance of the intercellular interference power upper limit is calculated as:

$$
\begin{aligned}
\operatorname{var}[P]_{\text {out }, s}= & (1-\phi) N_{u, s} P_{r, s}^{2}\left(p \alpha_{s}-q \alpha_{s}^{2}\right) \\
& \times \sum_{j=1}^{N_{\text {cells }}-1} \int_{0}^{2 \pi} \int_{0}^{R}\left(10 \frac{G\left(\psi_{o, i j}\right) G\left(\psi_{i j}\right)}{10}\right)^{2} \frac{1}{\pi R^{2}} r_{i j} d r_{i j} d \theta_{i j}
\end{aligned}
$$

The variance of the total interference power of the service $s$ is given by:

$\operatorname{var}[P]_{t, s}=\operatorname{var}[P]_{\text {in,s }}+\operatorname{var}[P]_{\text {out }, s}$

The variance of the total interference power due to the $M$ services is given by:

$\operatorname{var}[P]_{t}=\sum_{s=1}^{M} \operatorname{var}[P]_{t, s}$

Taking into account that about (14/16 to $15 / 16=\varepsilon)$ of the received power $P_{r, s}$ is used in the detection [13], the relation carrier to interference $(\mathrm{C} /)_{s}$ of the service $s$ is given by:

$\left[\frac{C}{I}\right]_{s}=\frac{\varepsilon P_{r, s}}{E[P]_{t}+P_{N}+\gamma \sqrt{\operatorname{var}[P]_{t}}}$

Where $P_{N}$ is the receiver noise power and $\gamma$ is a factor that depends on the outage probability ( 2.05 for outage probability of $2 \%$ and it is 2.33 for an outage probability of $1 \%$ ).

Then, the $\left(E_{b} / N_{o}\right)_{s}$ ratio is given as:

$\left[\frac{E_{b}}{N_{o}}\right]_{s}=\left[\frac{C}{I}\right]_{s} G_{p, s}$

Where $G_{p, s}$ is the processing gain of the service $s$.

The received power $P_{r, s}$ of the service $s$ is given by:

$P_{r, s}=P_{t, s}+G_{T X}+G_{R X}-L_{p}$

Where $P_{t, s}$ is the maximum transmitted power of the user of the service $s$ when it is at the border of the cell under study, $G_{T X}$ is the antenna gain of the mobile assumed to be $0 \mathrm{~dB}, G_{R X}$ is the antenna gain of the HAP in the direction of the user and $L_{p}$ is the propagation loss given by:

$L_{p}=20 \log _{10}\left(\frac{4 \pi d}{\lambda}\right)+L_{\text {Shad }}+L_{\text {walls }}$
Where $d$ is the distance in $\mathrm{m}$ between the user and the HAP, $\lambda$ is the wavelength, $L_{\text {Shad }}$ is the shadowing margin assumed to be $8 \mathrm{~dB}$ and $L_{\text {walls }}$ is the walls penetration (entry) loss for indoor users.

For mixed services of voice and data, the data user maximum transmitted power to the maximum transmitted power of the voice users given in decibels should be:

$$
\left(\frac{P_{t, d}}{P_{t, v}}\right)_{d B}=(1+\delta) \quad\left[10 \log _{10}\left(\frac{G_{p v} /\left(\frac{E_{b}}{N_{o}}\right)_{v}}{G_{p d} /\left(\frac{E_{b}}{N_{o}}\right)_{d}}\right)\right]
$$

where

- $P_{i, d}$ is the transmitted power of the data users the exists in the cell border;

- $P_{t, v}$ is the transmitted power of the voice users the exists in the cell border;

- $\delta$ is a constant with a value of 0.0 if only the mean value of the interference is considered. When the interference variance is also considered, it has a value of $(-0.1$ to 0.1$)$ depending on the parameters of the services under study.

- $G_{p v}$ is the voice service processing gain;

- $G_{p d}$ is the data service processing gain;

- $\left(E_{b} / N_{o}\right)_{v}$ is the required $\left(E_{b} / N_{o}\right)$ for voice service given in real numbers; and

- $\left(E_{b} / N_{o}\right)_{d}$ is the required $\left(E_{b} / N_{o}\right)$ for data service given in real numbers.

If the adjacent channel interference (ACI) is considered, then the new expected value and the variance of total interference are given by:

$E\left[P_{t}\right]_{\text {new }}=E[P]_{t} \quad\left[1+k_{1}\right.$ ACLR $+k_{2}$ ACLR $]$

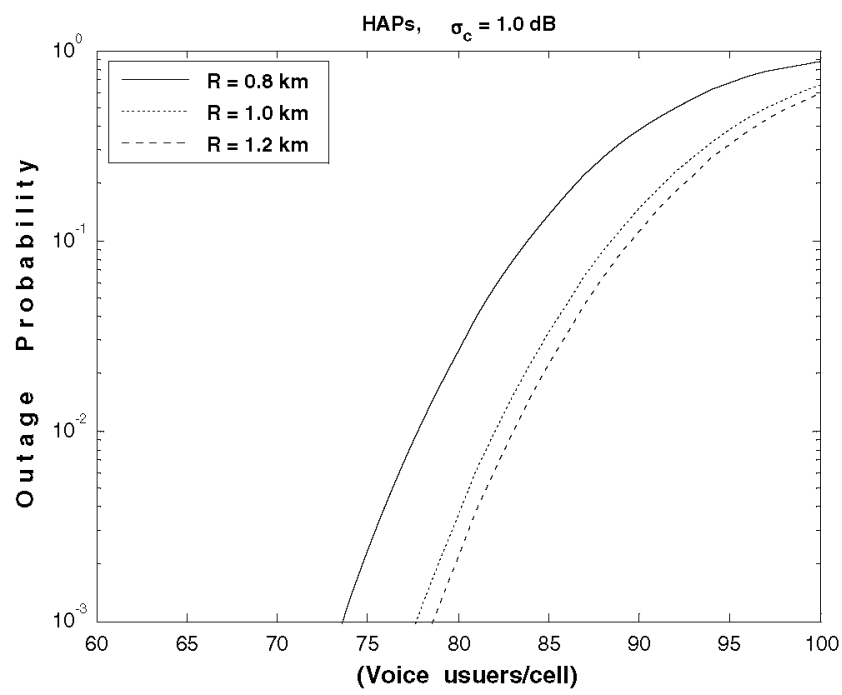

Fig. 3 Uplink performance for outdoor voice users 


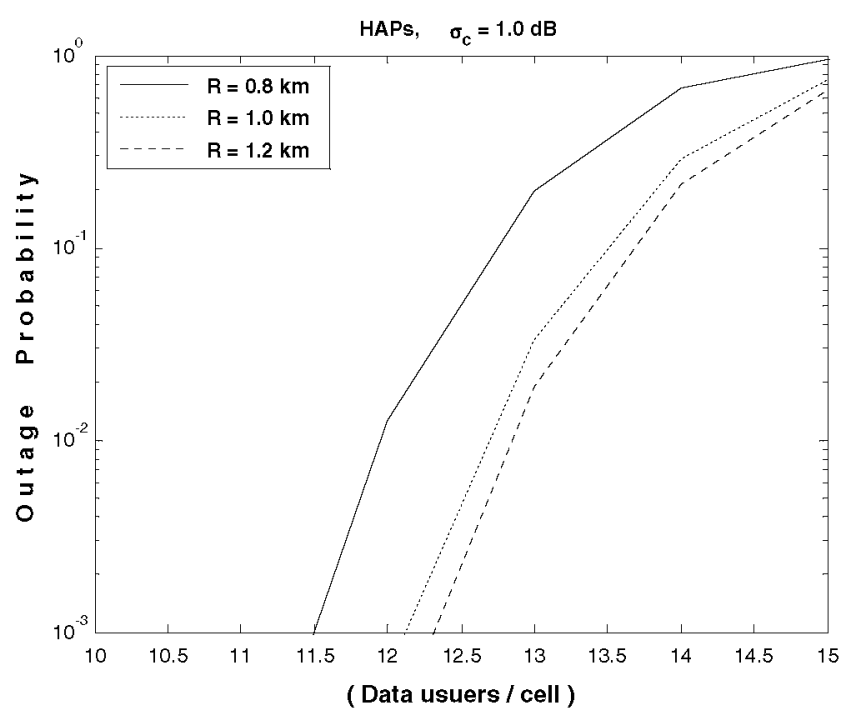

Fig. 4 Uplink performance for outdoor data users

$\operatorname{var}\left[P_{t}\right]_{\text {new }}=\operatorname{var}[P]_{t} \quad\left[1+k_{1}^{2}(\mathrm{ACLR})^{2}+k_{2}^{2}(\mathrm{ACLR})^{2}\right]$

where $k_{1}$ and $k_{2}$ are the interference factors due to the lower and higher adjacent channel, respectively, and ACLR is the adjacent channel leakage ratio with a typical value of -30 to $-40 \mathrm{~dB}$. For collocated HAPs, $k_{1}$ and $k_{2}$ have the value of 1 .

\section{Numerical results}

We assume a platform altitude of $h=20 \mathrm{~km}, \varepsilon=15 / 16$, $\mathrm{ACLR}=-40 \mathrm{~dB}(0.0001), k_{1}=k_{2}=1$ and an imperfect power control with $\sigma_{\mathrm{c}}=1.0 \mathrm{~dB}$ unless other values are mentioned. Frequency of operation is assumed to be $1,950 \mathrm{MHz}$. We

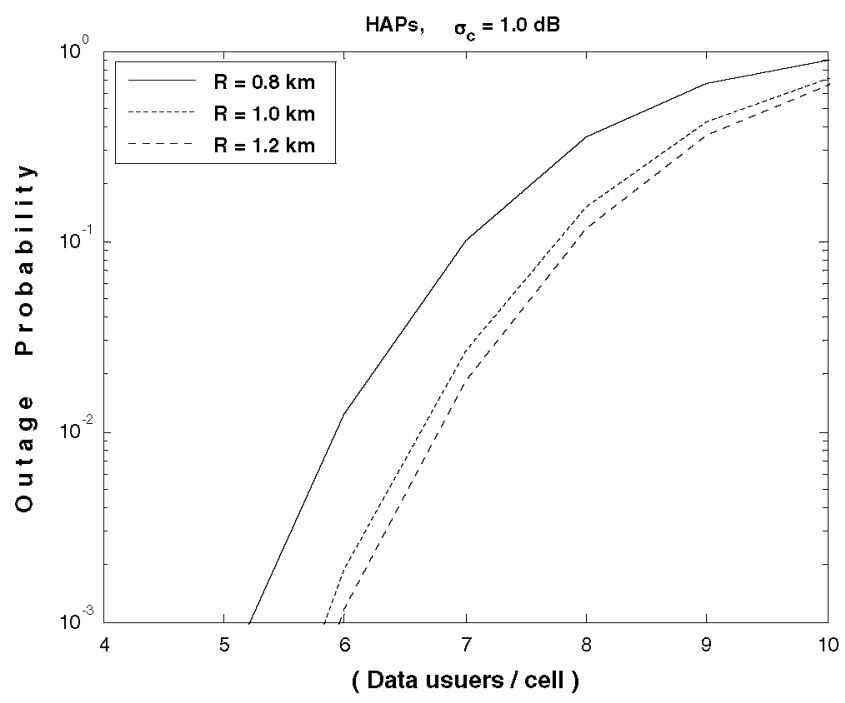

Fig. 5 Uplink performance for outdoor data users and 40 outdoor voice users/cell

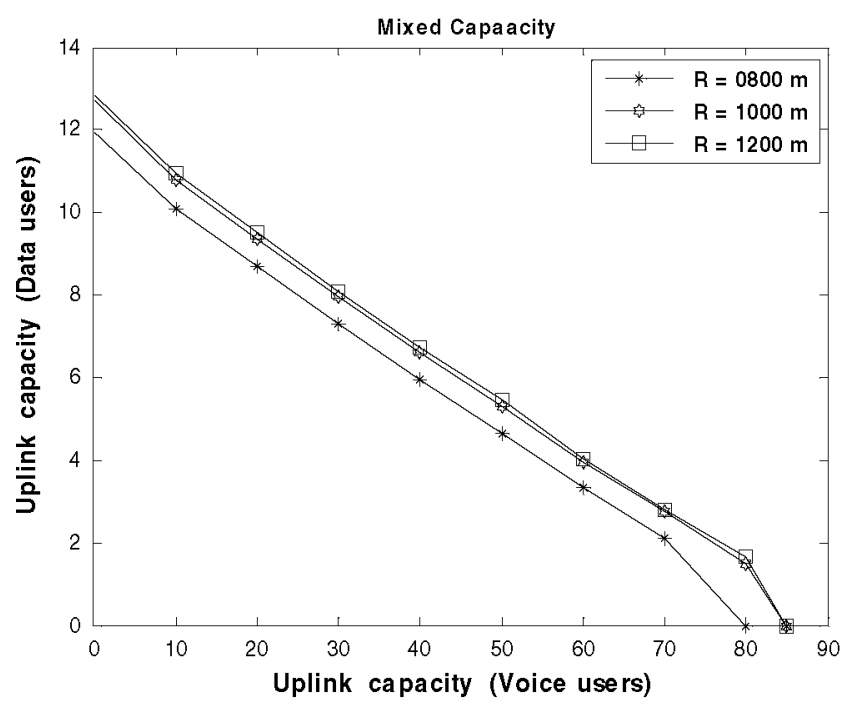

Fig. 6 Uplink mixed capacity for outdoor users

will assume that the cell capacity is the number of users when the outage probability is $1 \%$.

We first study the case of voice users only assuming that [13]:

- $G_{p v}=256$;

- $\left(E_{b} / N_{o}\right)_{v}=5 \mathrm{~dB}$;

- $\alpha_{v}=0.67$;

- $P_{t, v}=23 \mathrm{dBm}$.

Figure 3 shows the uplink performance for three different cell radii. It can be noticed that the uplink capacity is 77.6 , 82.0 and 83.0 outdoor voice users for cell radius of 800 , 1,000 , and $1,200 \mathrm{~m}$, respectively. The increment of the uplink capacity with the increment of the cell radius is due to the fact that the intercellular interference is lower for

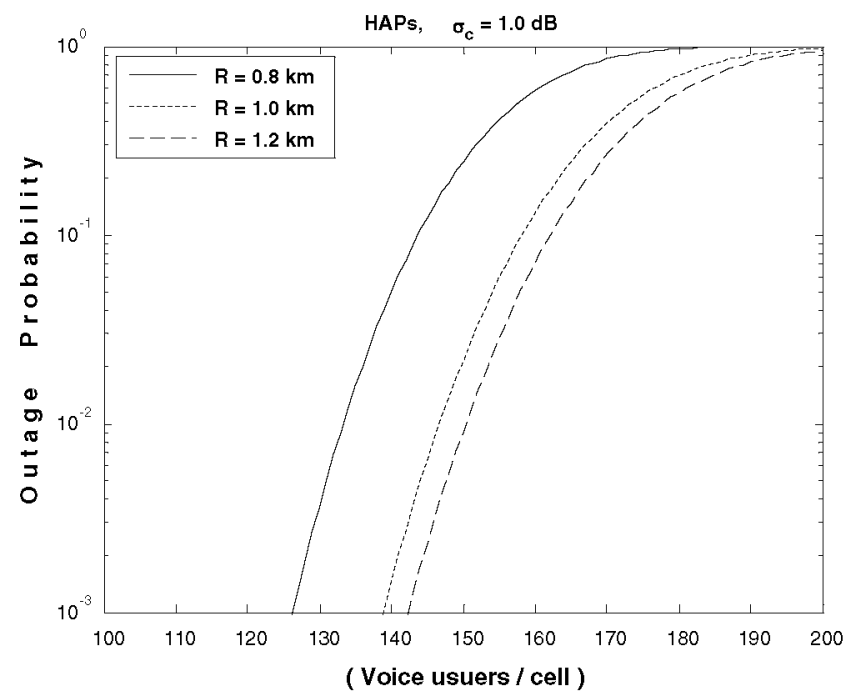

Fig. 7 Uplink performance for outdoor voice users only when synchronous WCDMA is used 


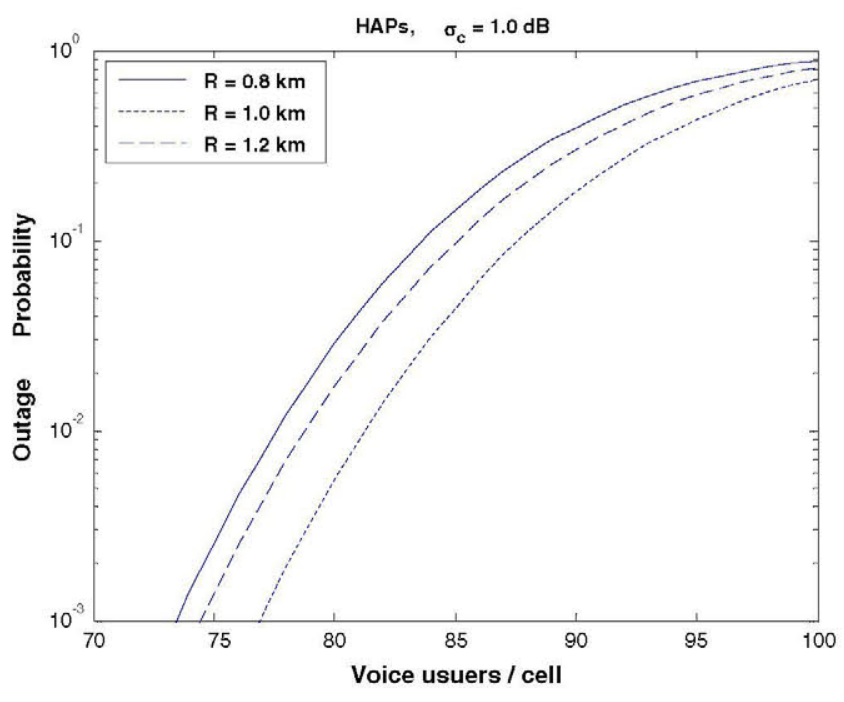

Fig. 8 Uplink performance for indoor voice users

higher cell radius. For a cell radius higher than $1,200 \mathrm{~m}$, the intercellular interference remains almost constant meanwhile the received signal will be lower due to the lower gain of the receiving antenna. Thus, increasing the cell radius will reduce the uplink capacity.

Let us now study the case of data users only assuming the following typical values [13]:

- $G_{p d}=32$;

- $\left(E_{b} / N_{o}\right)_{d}=2.5 \mathrm{~dB}$;

- $\alpha_{d}=1$;

- $P_{t, d}=30 \mathrm{dBm}$.

Figure 4 shows the uplink performance for three different cell radii. It can be noticed that the uplink capacity is 11.95 , 12.7 and 12.85 outdoor data users for cell radius of 800 , 1,000 , and $1,200 \mathrm{~m}$, respectively.

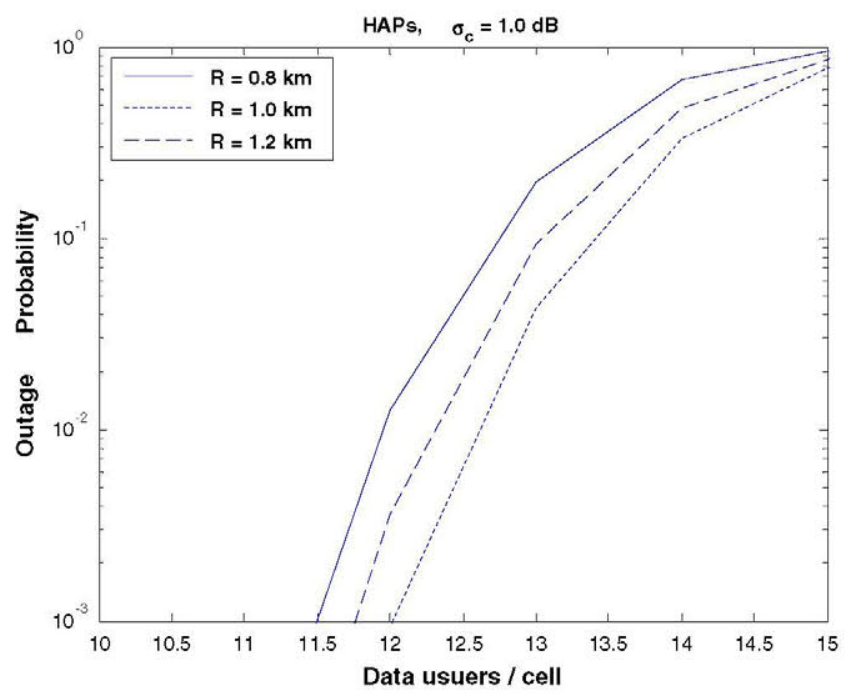

Fig. 9 Uplink performance for indoor data users

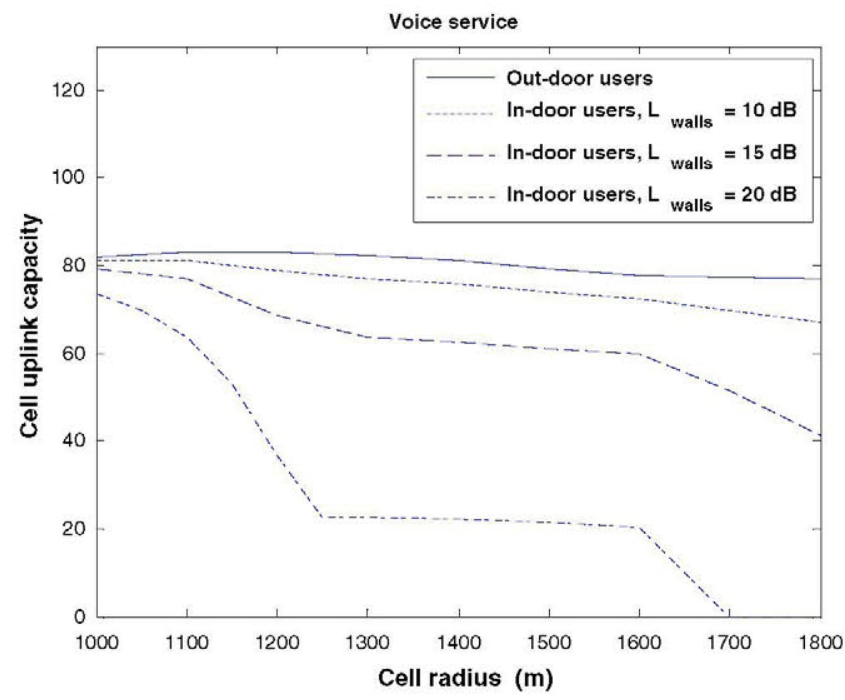

Fig. 10 Cell uplink capacity as a function of the cell radius for voice users

Let us now study the case of mixed services. Figure 5 shows the uplink performance for three different cell radii assuming 40 outdoor voice users within each cell. It can be noticed that the uplink extra capacity is 5.9, 6.6 and 6.8 outdoor data users for cell radius of $800,1,000$, and $1,200 \mathrm{~m}$, respectively. This shows that increasing the number of the voice users within the cell will reduce the data user's capacity.

Figure 6 shows the mixed capacity of the WCDMA HAP system. It can be noticed that the relation between the voice service uplink capacity and the data service uplink capacity is almost linear.

Figure 7 shows the uplink performance for three different cell radii when synchronous WCDMA is used $(\phi=0.5)$. It can be noticed that the uplink capacity is $133.2,146.6$ and 150.2 outdoor voice users for cell radius of $800,1,000$, and

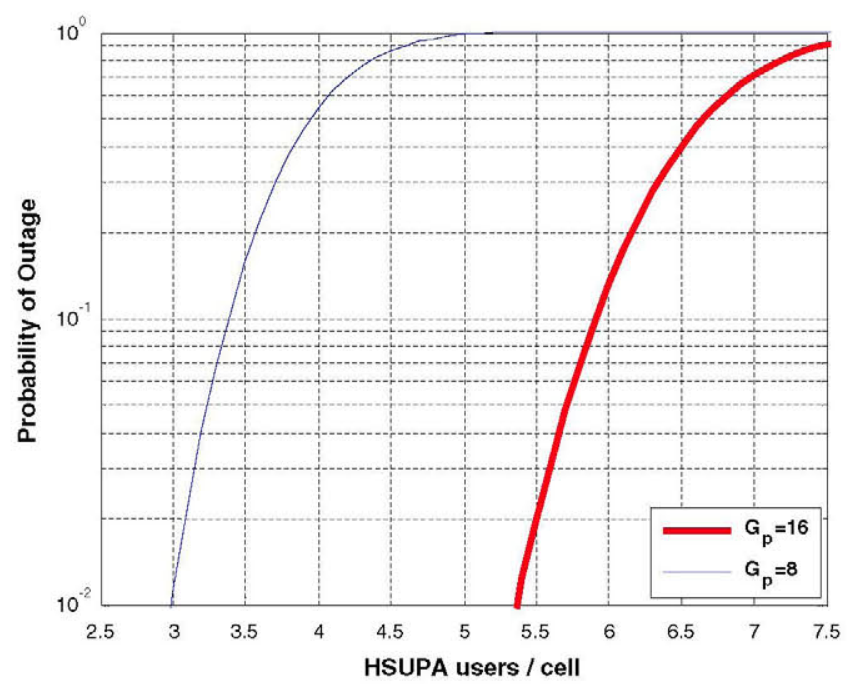

Fig. 11 Uplink performance for indoor HSUPA users 
$1,200 \mathrm{~m}$, respectively. Comparing the results of this figure with the results of Fig. 3, it can be noticed that the capacity here is higher than the capacity given by Fig. 3 when asynchronous WCDMA is used. This is because of the lower intracellular interference.

Figure 8 represents the uplink performance for three different cell radii when one or more than one of the voice service users is indoor voice users. It can be noticed that the uplink capacity is $77.5,79.3$ and 78.7 voice users for cell radius of $800,1,000$, and $1,200 \mathrm{~m}$, respectively. Comparing these capacities with those given by Fig. 3, it can be noticed that the uplink capacity for an indoor users is lower than the capacity for an outdoor users. This is due to the higher propagation loss that reduces the power of the received signal at the base station. Also, it can be noticed that the uplink capacity at a cell radius of $1,200 \mathrm{~m}$ is lower than that at a cell radius of $1,000 \mathrm{~m}$. This is due to the lower power received in the base station which cannot be compensated by the lower value of the intercellular interference.

Figure 9 depicts the uplink performance for three different cell radii when one or more than one of the data service users is indoor data users. It can be noticed that the uplink capacity is $11.9,12.6$ and 12.3 data users for cell radius of $800,1,000$, and $1,200 \mathrm{~m}$, respectively.

Figure 10 illustrates the uplink capacity as a function of the cell radius for outdoor and indoor users with three different values of $L_{\text {walls }}$ [14]. For the outdoor user's case, it can be noticed that for a cell radius higher than $1,200 \mathrm{~m}$, the uplink capacity reduces with the increment of the cell radius. This is due to the fact that increasing the cell radius will reduce the power of the received signal due to the reduction of the receiving antenna gain which cannot be compensated by the reduction of the intercellular interference. For the indoor user's case, it can be noticed that increasing the wall's penetration loss reduces the uplink capacity due to the lower power of the received signal. It can be concluded that indoor users can be supported with a reasonable capacity when the cell radius is $1,000 \mathrm{~m}$, whatever the walls penetration loss is. It can be noticed that for walls entry loss of $20 \mathrm{~dB}$, the uplink capacity will be null for a cell radius of $1,700 \mathrm{~m}$ or more.

For a HAP system with 37 beams and a cell radius of $1,000 \mathrm{~m}$, the total uplink capacity of the system will be 3,034 outdoor voice users or 444 outdoor data users. When one or more than one user is an indoor user, the uplink capacity is 2,923 voice users or 444 data users for $L_{\text {walls }}$ of $10 \mathrm{~dB}$.

Let us study the case when the HSUPA service is supported in the uplink. Figure 11 shows the uplink capacity will be 5.35 HSUPA users with a processing gain of 16 or 3.0 HSUPA with a processing gain of 8 assuming a required $\mathrm{E}_{\mathrm{b}} / \mathrm{N}_{\mathrm{o}}$ of $4.8 \mathrm{~dB}$. This is the case when there are no HSUPA users in the cells around the one under study. In contrary, the cell uplink capacity will depend on the locations of the HSUPA users within other cells.
If the ACLR is increased to $-30 \mathrm{~dB}(0.001)$, the uplink capacity will be almost $99.8 \%$ of each one of the abovementioned capacities. Without the adjacent cell interference, capacity will be only $0.02 \%$ higher than the abovementioned capacities.

\section{Conclusions}

In this work, the capacity and the interference statistics of the uplink of HAPs WCDMA with an imperfect power control have been investigated. The uplink capacity has been given for different cell radius assuming voice and data users. For 37 cells HAP, the total uplink capacity is 3,034 outdoor voice users or 444 outdoor data users. When one or more than one user is an indoor user, the uplink capacity is 2,923 voice users or 444 data users for $L_{\text {walls }}$ of $10 \mathrm{~dB}$. It has been shown that the effect of the adjacent channels interference is very small.

\section{References}

1. Djunnic GM, Freidenfelds J (1997) Establishing wireless communications services via high-altitude aeronautical platforms: a concept whose time has come? IEEE Commun Magazine 35(9):128-135

2. Gejji RR (1992) Forward-link power control in CDMA cellular systems. IEEE Trans Veh Technol 41:532-536

3. Foo YC, Lim WL, Tafazolli R, Barclay LW (2001) Forward link power control for high altitude platform stations W-CDMA system. IEEE VTC 2001, pp. 625-629, Fall 2001

4. Ahmed BT, Ramón MC, Ariet LH (2003) On the downlink capacity of high altitude platform W-CDMA system. IST 2003, Aviero, Portugal, pp. 765-768

5. Foo YC, Lim WL, Tafazolli R, Barclay L (2000) Other-cell interference and reverse link capacity of capacity of high altitude platform station CDMA system. IEE Electron Lett 36:1881-1882

6. Karapantazis S, Pavlidou FN (2005) The role of high altitude platforms in beyond $3 \mathrm{G}$ network. IEEE Wirel Commun 12(6):33-41

7. Karapantazis S, Pavlidou FN (2007) Call admission control in multiservice high altitude platform (HAP) W-CDMA cellular systems. Comput Netw 51(12):3491-3506

8. Karapantazis $S$, Pavlidou FN (2005) Impact of imperfect power control multiuser detection on the uplink of a WCDMA high altitude platform system. IEEE Commun Lett 9(5):414-416

9. Hong TC, Ku BJ, Park JM, Ahn DS, Jang YS (2006) Capacity of the WCDMA system using high altitude platform station. Int J Wirel Inf Netw 13(1):5-17

10. Carnero JO, Pedersen KI, Mogensen PE (2004) Capacity gain of an uplink-synchronous WCDMA system under channelization code constraints. IEEE Trans Veh Technol 53(4):982-991

11. Jansen MG, Prasard R (1994) Capacity, throughput, and delay analysis of a cellular DS CDMA system with imperfect power control and imperfect sectorization. IEEE Trans Veh Technol 44(1):57-75

12. Romero-Jerez JM, Tellez-Labao C, Diaz-Estrella A (2004) Effect of power control imperfections on the reverse link of cellular CDMA networks under multipath fading. IEEE Trans Veh Technol 53(1):61-71

13. Holma H, Toskla A (2002) WCDMA for UMTS, 2nd edn. Wiley, New York

14. Axiotis DI, Theologou ME (2003) An empirical model for predicting building penetration loss at $2 \mathrm{GHz}$ for high elevation angles. IEEE Antenna Wirel Propag Lett 2:234-237 